\title{
Oferta y Potencial de Desarrollo de Atributos de Valor de Productos Cárnicos Bovinos en el Mercado Minorista Chileno.
}

\author{
Claus Köbrich $^{1}$, Carolina Farías ${ }^{1}$, Mario Maino ${ }^{1}$ \\ ${ }^{1}$ Departamento de Fomento de la Producción Animal, Facultad de Ciencias Veterinarias y Pecuarias \\ Universidad de Chile. \\ Email: ckobroch@uchile.cl
}

\begin{abstract}
Resumen
A pesar de la fuerte competencia y los importantes cambios que se han producido en el mercado de las carnes en las últimas décadas, las características de la oferta de carne bovina no han presentado grandes cambios. Este estudio analizó la oferta del sector minorista para describir el desarrollo cualitativo de los productos, identificando atributos diferenciadores presentes en supermercados, carnicerías y distribuidores en línea de carne bovina chilenos, así como la oferta de carne bovina de calidad en Estados Unidos, Reino Unido, Francia y España mediante una revisión en línea. Luego se entrevistó a actores del sector minorista de carne, para conocer su opinión acerca del desarrollo de productos diferenciados en el mercado nacional y sus limitaciones. Los resultados mostraron que el desarrollo de este mercado es todavía escaso. Nuevas presentaciones para un mismo corte, un sistema nacional de graduación de la carne, carne magra y platos preparados son algunas oportunidades a corto plazo que podrían ser atractivas para la totalidad de la cadena de la carne. Sin embargo, para que estos productos sean exitosos es fundamental desarrollar marcas, educar e informar a los consumidores y articular la cadena de la carne bovina nacionaltécnicas de evaluación y mejora del bienestar, entre otras que promuevan la reintroducción de individuos rehabilitados.
\end{abstract}

Palabras clave: Carne bovina, atributos de calidad, percepción de calidad, actitud de los consumidores, Chile.

\section{Introducción}

Los productos cárnicos participan en un mercado muy competitivo, que ha sufrido grandes cambios en los últimos años. Si bien la industria de aves y cerdos ha ampliado considerablemente su oferta a través de la agregación de valor, el subsector de carne bovina no se ha desarrollado de la misma manera. Entre las razones que explicarían esta situación están la escasa articulación de esta cadena productiva, el mayor costo de producción en relación a otras carnes, el incremento en las importaciones, el descenso en su consumo, la disminución en la producción de carne en vara y los cambios en las preferencias de los consumidores (SAGIICA, 1999; Fundación Chile, 2000; PROCOM, 2004; ODEPA, 2007). Dado que en Chile los precios internos de la carne están muy influidos por los internacionales y que en este rubro existe poco espacio para disminuir costos, las miradas se han puesto sobre las opciones para mejorar la calidad de la oferta chilena. La razón de esto es que al tomar decisiones de compra, los consumidores consideran principalmente el precio y la calidad del producto (Grunert, 1997; Becker y col.,2000; Bernués y col.,2003).Distintos investigadores han intentado explicar el proceso de percepción de calidad (Becker, 2000; Grunert y Bech Larsen, 2004; Brunsøy col.,2005). Este proceso comienza cuando el consumidor observa el producto y recibe información de sus características, creando expectativas respecto a su calidad. Esta información se recibe a través de señales de calidad, que pueden ser intrínsecas o extrínsecas (Grunert y Bech Larsen, 2004). Las señales intrínsecas se relacionan con aspectos físicos del producto, como la cantidad de grasa de la carne, por ejemplo. Las extrínsecas no son físicamente parte del producto, por lo que se aplican externamente, como los envases. Sólo algunas de las señales de calidad que emite un producto, son percibidas por el consumidor para crear una expectativa de calidad. 
Las señales que el consumidor integra provienen de las características del producto que son valoradas al momento de elegir, características que se conocen como atributos de calidad (Pride y Ferrell, 1997; Becker y col., 2000; Bernués y col.,2003). Dependiendo del autor y del punto de vista, los atributos de calidad se han clasificado de distintas maneras. Al igual que las señales de calidad, los atributos pueden clasificarse según su naturaleza, como intrínsecos o extrínsecos. Los primeros están asociados directamente al producto, mientras los segundos no se asocian directamente a él (Brunsøy col., 2005). Según el momento en el que son percibidos, los atributos pueden clasificarse como de búsqueda, de experiencia o de confianza. Los atributos de búsqueda, se evalúan durante la decisión de compra, como por ejemplo, la marca. Los atributos de experiencia, sólo pueden evaluarse después de consumir el producto, como la terneza de la carne, por ejemplo. Los atributos de confianza, como ser orgánico, no pueden ser reflejados físicamente en el producto (Grunert, 1997). Por esta razón, los atributos de confianza deben contar con programas de certificación de calidad, que aseguren el cumplimiento del atributo. Una forma como el producto muestra que ha sido certificado, es mediante la presencia de un sello en el envase, que permite que un consumidor informado lo diferencie de otros similares (Oyarzún, 2001; CE, 2005). Esta calidad observada y evaluada por el consumidor, es muy subjetiva y dinámica en el tiempo, ya que se asocia a una serie de elementos estrechamente relacionados con el consumidor (aspectos sociales, económicos y culturales) y el producto (características físicas, procesos productivos y controles de calidad) (Grunert y col., 1996; Becker, 2000; Brunsøy col., 2005). La teoría económica establece que, cuando aumenta el poder adquisitivo de una población y sus necesidades básicas son satisfechas, se inicia la búsqueda de nuevos atributos de calidad (Becker, 2000; Oyarzún, 2001; Grunert y col., 2002). Este sería el caso de una proporción de los consumidores nacionales, que valoran un producto de calidad y están dispuestos a pagar un sobreprecio por él (Fundación Chile, 2000). Aunque este escenario constituye una oportunidad para la agregación de valor y los especialistas coinciden en que la diferenciación es la clave para acceder a mercados extranjeros, no se conoce bien la presencia de carne bovina diferenciada en el mercado interno. Frente a la ausencia de estudios concernientes a este tema, este trabajo planteó como objetivo general analizar la oferta nacional de carne bovina a nivel minorista y los factores que afectan su desarrollo. Los objetivos específicos a cumplir fueron: a) identificar los atributos de valor presentes en la carne bovina de la oferta minorista del país; b) identificar atributos de valor para nuevos productos cárnicos bovinos diferenciados; c) conocer la opinión del sector minorista respecto a la situación actual de su oferta, sus limitaciones y tendencias; d) proponer atributos posibles de desarrollar en nuestro mercado y algunos elementos estratégicos para lograrlo.

\section{Materiales y métodos}

La investigación tuvo tres fases, realizadas en el año 2009 en Santiago de Chile. La primera fase identificó los atributos de calidad presentes en la carne bovina del segmento minorista nacional. Para ello se realizó una investigación cualitativa de tipo exploratorio en supermercados, carnicerías y distribuidores minoristas de carne a pedido que abastecen el sector oriente de la Región Metropolitana con carne bovina fresca, refrigerada o congelada, ya sea en trozo, cubos, molida y laminada. La recolección de datos en supermercados y carnicerías se hizo por medio de visitas. En el caso de las distribuidoras a pedido, se recolectó la información entregada por sus sitios web, apoyada con entrevista telefónica cuando fue necesario. Se registró todo atributo detectable por el consumidor al momento de la decisión de compra. Dicho procedimiento se realizó dos veces en cada punto de venta por la misma persona, separados por un período aproximado de tres meses.

En la segunda fase, se buscó atributos desarrollados en otros países y que no existieran o tuvieran escaso desarrollo en nuestro mercado, a través de una investigación cualitativa exploratoria en sitios web de supermercados, carnicerías y distribuidoras de carne a pedido de Estados Unidos, Reino Unido, Francia y España. Los datos recolectados en 75 empresas, fueron tabulados, categorizados y analizados de la misma forma que los obtenidos en el mercado nacional.

La tercera fase tuvo por fin de conocer la opinión de expertos del sector, respecto a los atributosencontrados y al potencial de desarrollo de productos diferenciados en nuestro mercado. Para ello se aplicó una entrevista semi-estructurada a expertos de cadenas de 
supermercados (4), carnicerías (3), ligados a instituciones o empresas del sector (3), representantes de distribuidoras (3) y 1 productor de carne bovina diferenciada. La entrevista tuvo preguntas abiertas, relativas a las nuevas tendencias observadas al momento de realizar la compra de carne, la percepción de los entrevistados respecto a estas nuevas preferencias, los atributos de calidad posibles de desarrollar en nuestro mercado y las limitaciones asociadas a ello. Las entrevistas se registraron mediante grabaciones o notas escritas. Las preguntas se desarrollaron de forma tal, que cada una de ellas apuntara hacia un concepto clave o categoría a analizar, como sugiere la técnica de análisis cualitativo conocida como Teoría Fundamentada. Dicha técnica consiste en la identificación de conceptos entregados por los entrevistados, en forma de temas, categorías y subcategorías, para luego organizarlas en un modelo de interrelaciones que representará la teoría que explica el fenómeno en estudio (Sampieri y col., 2006).

Por último, a partir de estos resultados se propone atributos posibles de desarrollar en nuestro mercado y algunos elementos estratégicos para lograrlo.

\section{Resultados y Discusión}

\subsection{Atributos presentes en los mercados}

Los atributos encontrados en 23 puntos de venta, correspondientes a 16 empresas, se agruparon en siete categorías que definen una misma propiedad en el producto final.

Cuadro 1: Categorías de análisis para atributos de calidad presentes en Chile y el extranjero.

\begin{tabular}{|c|c|c|c|}
\hline Categoría & Concepto & Atributo de calidad & Ejemplos \\
\hline $\begin{array}{l}\text { Calidad } \\
\text { Sensorial }\end{array}$ & $\begin{array}{l}\text { Productos que se diferencian sensorialmente } \\
\text { al momento de ser consumidos (terneza, } \\
\text { jugosidad, sabor) }\end{array}$ & $\begin{array}{l}\text { Raza, certificación de } \\
\text { origen, graduación de } \\
\text { carnes, sello de excelencia, } \\
\text { maduración }\end{array}$ & $\begin{array}{l}\text { Angus, Wagyú, Label } \\
\text { Rouge, Dry Aged, } \\
\text { USDA Beef Grading }\end{array}$ \\
\hline Conveniencia & $\begin{array}{l}\text { Presentaciones del producto que facilitan su } \\
\text { preparación o almacenamiento. }\end{array}$ & $\begin{array}{l}\text { Preparación previa, } \\
\text { envasado conveniente }\end{array}$ & $\begin{array}{l}\text { Carne aliñada, } \\
\text { carpaccio, envase apto } \\
\text { para microondas }\end{array}$ \\
\hline Presentación & $\begin{array}{c}\text { Formas físicas en las que se puede presentar } \\
\text { un corte de carne para diferenciarlos de los } \\
\text { productos genéricos. }\end{array}$ & Corte de fantasía & Arrachera, baseball \\
\hline Marca & $\begin{array}{c}\text { Término o diseño que identifica los productos } \\
\text { de un vendedor y los diferencia de los } \\
\text { competidores. }\end{array}$ & Nombre de la marca & -- \\
\hline Saludable & $\begin{array}{l}\text { Características del producto que evitan } \\
\text { posibles daños a la salud del consumidor. }\end{array}$ & Natural, orgánico, magro & Carpaccio Magro \\
\hline $\begin{array}{l}\text { Tipo de } \\
\text { Animal }\end{array}$ & $\begin{array}{l}\text { Tipo de animal desde el cual se obtuvo el } \\
\text { producto, definido según su edad fisiológica y } \\
\text { sexo. }\end{array}$ & Ternera, buey & $\begin{array}{l}\text { Ternera lechal, buey } \\
\text { gallego }\end{array}$ \\
\hline Religión & $\begin{array}{l}\text { Procedimientos realizados durante el proceso } \\
\text { productivo con fines religiosos. }\end{array}$ & & Kosher, halal \\
\hline
\end{tabular}


La categoría calidad sensorial incluye cinco tipos de atributos. El primero es raza y se observó en Chile seis empresas que la explicitan en el envase, el punto de venta o el sitio web de compra. En otros países, destaca EEUU donde es frecuente que empresas mencionen la raza como atributo, en particular Angus, Hereford y Wagyú. Incluso existe programa Certified Angus Beef $\AA$, creado por la Asociación de Angus Americano con el fin de asegurar sabor, terneza y jugosidad en sus productos, y que en la actualidad vende alrededor de 2,7 mil millones de dólares al año (CAB, 2010). En los países europeos se ofrece además otras razas como Charolaise, Limousine y Normando. El segundo atributo es la certificación de origen que reconoce la mejor palatabilidad de debida a factores naturales y humanos asociados a una zona geográfica específica (Loureiro y McClaskey, 2000; CE, 2007). En Chile no se encontró productos con estas características, a pesar de existir un marco regulatorio (Ley de Propiedad Industrial, 19.996 de 2005) que distingue Indicación Geográfica (IG) y Denominación de Origen (DO). En Estados Unidos se encontró productos que dicen provenir de regiones con buena reputación en cuanto a carne bovina de calidad, pero ninguna bajo algún sello que lo certifique. Síse encontró productos bajo el programa de certificación de origen de la Unión Europea, como por ejemplo la Indicación Geográfica Protegida (IGP) carne de Ternera Asturiana ${ }^{\circledR}$, que en 2009 vendió más de 5.000 toneladas de su línea tradicional y unas 150 toneladas de carne ecológica (Ternera Asturiana, 2010). El tercer atributo se refiere a sistemas de graduación, que a través de la medición de parámetros en el músculo predice su palatabilidad. El sistema de tipificación existente en Chile tipifica las canales en caliente y no los cortes o la carne (Ponce, 2005), por lo que no se ofrece en propiedad carnes con estos atributos. Estados Unidos cuenta con un sistema de categorización de carnes que diferencia ocho grados de calidad basados en la edad fisiológica del animal y en el marmoleo y color de la carne. El cuarto atributo es un sello que asegure la calidad sensorial; sin embargo, no se encontró algún sello asociado a un programa de certificación que explicite calidad superior. En Europa el sello Label Rouge, regulado por el Instituto Nacional Francés de Origen y Calidad, asegura al consumidor una calidad siempre superior a otros productos de similar naturaleza (Label Viande, 2010), ya que han sido producidos baja estándares superiores. Por ejemplo el Veau d'Aveyron et du Ségala, es carne de ternera de Limousine o Blonde d'Aquitaine, producida bajo estrictos estándares en cuatro departamentos de la región de Ségala. El último atributo sensorial encontrado fue la maduración, entendida como el almacenamiento de carne fresca a bajas temperaturas para mejorar su terneza mediante procesos enzimáticos y bioquímicos naturales que rompen los enlaces de tejido conectivo (Tatum y col.,1999; Lasta, 2003; RKM, 2010). El método tradicional consiste en mantener la carne a temperaturas de refrigeración por hasta 12 o 14 días. El madurado en seco o Dry Aged, en cambio, la mantiene a temperaturas entre 0 y $4^{\circ} \mathrm{C}$ por un periodo de 14 hasta 35 días, otorgándole mayor terneza y concentrando el sabor, debido a la evaporación de la humedad (Savell, 2008; Smith y col., 2008). Mientras en Chile toda la carne se ofrece madurada de manera tradicional, en locales especializados de Europa y Estados Unidos se encontró carnes de muy alto precio con madurado en seco (Dry Aged).

La conveniencia es la segunda categoría de atributos de calidad, esto es, productos que permiten reducir el tiempo y el esfuerzo utilizados para comprarlos, almacenarlos, prepararlos y consumirlos (Grunert y col.,2002). Respecto a productos preparados (no cocidos ni transformados), la oferta chilena es escasa. Incluye productos congelados, como brochetas o carpacho, y unos pocos refrigerados, como escalopas apanadas preparadas en los mismos puntos de venta o una marca que ofrecía porciones de carne marinada. En un sólo establecimiento se encontró preparaciones listas para cocinar (por ejemplo asiento de vacuno con filete de cerdo envuelto en espinacas). En el extranjero hay una gran variedad de estos productos. Sobre todo en carnicerías se ofrecen productos marinados, aliñados, saborizados, rellenos e incluso platos preparados a pedido, listos para calentar y servir. Además, se destaca una gran variedad de paquetes que contienen el corte de carne con los aliños necesarios para una receta o un conjunto de cortes parrilleros, prestablecidos o a elección. Esto último también se ofrece en algunas distribuidoras nacionales de carne a pedido. En cuanto al atributo de envasado, no se observó gran diferencia entre los distintos mercados. Sí se encontró opciones que facilitan la manipulación, almacenamiento e incluso la 
cocción del producto. Entre las innovaciones del mercado nacional para productos frescos se encontró bandejas de poliestireno aptas para su uso en microondas y entre los productos congelados, un sistema de bolsa resellable para churrascos, que vienen a su vez en bolsas de tres unidades cada una. Otros productos se ofrecían en cajas de cartón con vistosos diseños y colores, que contienen el producto envasado individualmente; y porciones individuales de carne molida congelada, envasadas en tripas de plástico corrugado. Cabe destacar los envases utilizados por algunas distribuidoras de carne extranjeras para el despacho a domicilio de sus productos, que cuentan con sistemas de control de temperatura y algunas hasta son envueltas elegantemente para entregar como regalo.

La categoría presentación, se refiere a los cortes cuyo nombre y músculos que la componen están normados de manera oficial (Ponce, 2005). Aunque es posible ofrecer cortes de fantasía que varían respecto a la norma (19.262 de 1992) se observó un escaso desarrollo de estos cortes en Chile. Sólo unas pocas empresas ofrecen cortes de fantasía, por lo general cortes típicos de otros países. En el extranjero, en cambio, se ofrece una gran variedad de cortes para elegir, con varias opciones para un mismo corte comercial, incluyendo con hueso, sin hueso o semi-deshuesado, variados tipos de recortes o despuntes, o la separación de la parte más noble de un corte comercial. Incluso se han realizado estudios para identificar músculos con atributos más deseados en la canal, para valorizarlos y agregar valor a la canal (Von Seggern y col., 2005; Calkins y col., 2005).

La marca, es una tributo en sí que también actúa como señal extrínseca, al transformar los atributos de confianza o experiencia en atributos de búsqueda (Grunert y col., 2002; Bredahl, 2003). La mayoría de los logos o imágenes asociados a marcas encontrados en Chile correspondía a productos congelados, como churrascos o carpachos. En la carne fresca se observó un esfuerzo por desarrollar marcas, ya que la mayoría de los supermercados ofrece al menos una de ellas. Incluso una cadena de supermercados desarrolló una marca propia con sellos prime, natural y orgánico y una planta faenadora una marca con carnicerías especializadas en productos de excelencia. En el caso de carnicerías o distribuidoras de carne a pedido el nombre de la empresa cumple la función de la marca. En el extranjero se encontró marcas que intentaban reflejar calidad y prestigio y en más de una ocasión un mismo establecimiento diversificaba su oferta con líneas tales premium, natural o vintage, por ejemplo.

La categoría saludable, definido en relación a nutrición e inocuidad del alimento (Grunert y col., 2002) presentó grandes diferencias. En Chile la oferta fue escasa, ya que sólo un supermercado y una carnicería ofrecían carne natural y el atributo magro sólo se encontró en carnes molidas, en Europa es frecuente encontrar el atributo orgánico y magro (o saludable), mientras que en EEUU se encuentran carnes naturales además de magras.

La categoría tipo de animal se refiere en Chile a las definiciones que emanan de la tipificación de las canales bovinas, basada en sexo, edad dentaria, grasa de cobertura, contusiones y, en el caso de los terneros, peso de la canal caliente. Diferente es en el extranjero, donde hay sistemas que diferencian el producto según el tipo de animal del cual proviene. En los países incluidos en este estudio, especialmente los europeos, se encontró una importante oferta de carne de ternera, que además es bastante diferenciada. En Chile en cambio, se encontró carne de ternera en un sólo establecimiento, que se confundía entre los otros cortes, al diferenciarse sólo por el etiquetado reglamentario. En algunos países europeos también fue se encontró carne de buey, producto que no se encontró en la muestra nacional.

La última categoría, religión, corresponde a productos obtenidos bajo los preceptos de la ley islámica (halal) o del judaísmo (kosher). Mientras en Chile sólo se encontró unas pocas marcas con productos kosher, en el exterior es común encontrar productos kosher (EEUU) y halal (Reino Unido).

\subsection{Potencial de desarrollo de productos diferenciados}

Las nuevas tendencias de los consumidores chilenos más mencionadas por los expertos fueron búsqueda de carne de razas especializadas, de gran calidad organoléptica o bajo contenido graso. Aunque $19 \%$ opinó que ello es una amenaza para el sector minorista, la gran mayoría (63 \%) las percibe como una oportunidad, reconociendo eso sí tres conjuntos de 
factores limitantes para el desarrollo del mercado de estos productos . El primero se refiere a restricciones de mercado, entre ellas el bajo volumen de comercialización de productos de calidad, los precios de este tipo de productos, el menor consumo relativo de carne bovina respecto a otras carnes y la escasa masa ganadera especializada. El segundo es la falta de una cultura cárnica, reflejada en el poco conocimiento que tienen los consumidores chilenos acerca de la calidad de la carne y los atributos que determinan la calidad. El tercer grupo son las normativas vigentes que impiden la agregación de valor y la diferenciación. Entre ellas la Norma de Tipificación de Canales y la ausencia de un sistema oficial de tipificación de carnes que permitiría generar una gradiente de precios, favoreciendo la comercialización de cortes de animales de calidad.

Figura 1: Nuevas tendencias reconocidas por los expertos en los consumidores de carne de alto poder adquisitivo en Chile ( $n^{\circ}$ de opiniones).

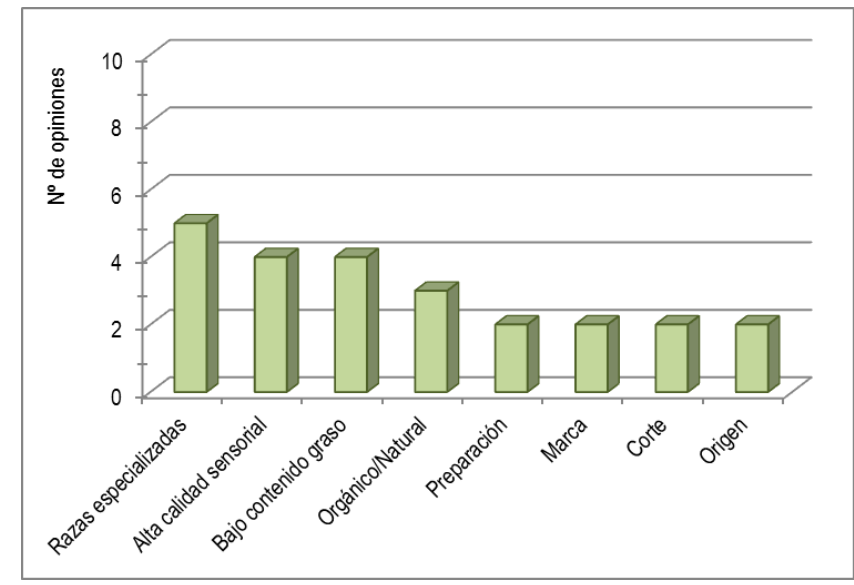

Figura 2: Limitaciones al desarrollo de productos diferenciados en el mercado nacional según los expertos ( $n^{\circ}$ de opiniones).

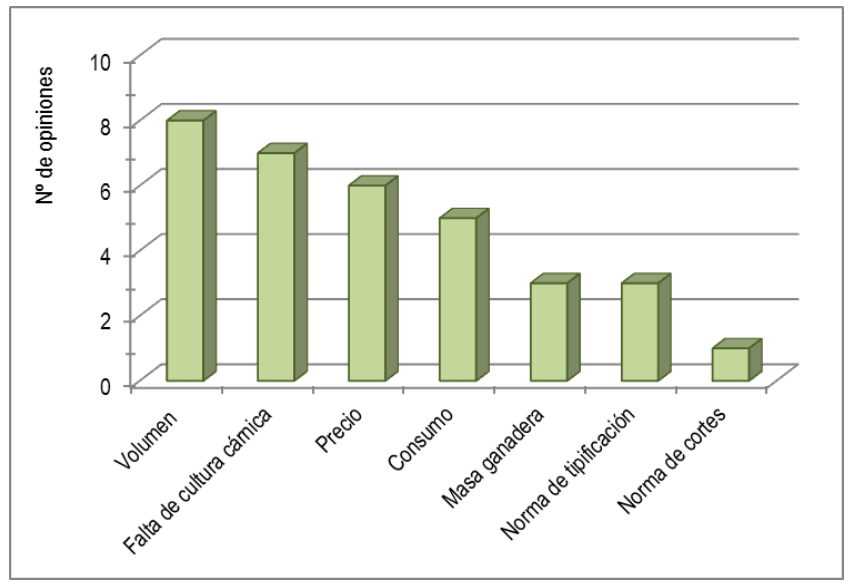

En cuanto a los atributos a desarrollar, mencionaron con más frecuencia conveniencia (carnes preparadas), calidad sensorial, saludable (magro u orgánico), y presentación (nuevos cortes). Como no es muy frecuente encontrar algún tipo de preparación en productos de carne bovina fresca en nuestro país, la mayoría de los entrevistados vio en ello una oportunidad, sosteniendo que productos más terminados serían exitosos en nuestro mercado. No se sugirió un mayor desarrollo de envases convenientes como un método de diferenciación potencialmente exitoso. Varios expertos coincidieron en que la carne sensorialmente superior, obtenida bajo altos estándares productivos, sería exitosa en el mercado interno. Sin embargo, pocos entrevistados sugieren el uso de razas especializadas, la denominación de origen, un sello de excelencia o el madurado en seco, como alternativas para lograrlo. Cuando se habla de un producto de calidad superior, casi siempre se refieren a un proceso productivo con altas exigencias a lo largo de todas sus etapas. Los expertos consideran además, que los productos saludables serían exitosos en nuestro mercado, en particular las carnes magras dado a la penetración que ha tenido la carne de pavo (percibida como muy magra). La opción de la carne orgánica sería en un plazo más largo, debido al pequeño nicho que lo demanda hoy en día. Tampoco descartaron la viabilidad futura de métodos productivos que no dejen residuos, no utilicen organismos genéticamente modificados y sean cuidadosos con el medioambiente y los animales. Los 
cortes de fantasía son uno de los atributos que parecen tener un amplio espacio de desarrollo. Entre las posibilidades se encuentra el desarrollo de cortes con hueso, asociados muchas veces a platos de fina presentación. Considerando la amenaza que constituyen los precios de la carne importada hacia el desarrollo de productos nacionales, los cortes con hueso son vistos además como una ventaja competitiva. El resto de los atributos identificados en las primeras etapas de este estudio se sugirieron aisladamente $o$ no fueron mencionadas como de interés por parte de los entrevistados.

Figura 3: Atributos de calidad identificados por los expertos como potencialmente exitosos en el mercado nacional ( $n^{0}$ de opiniones).

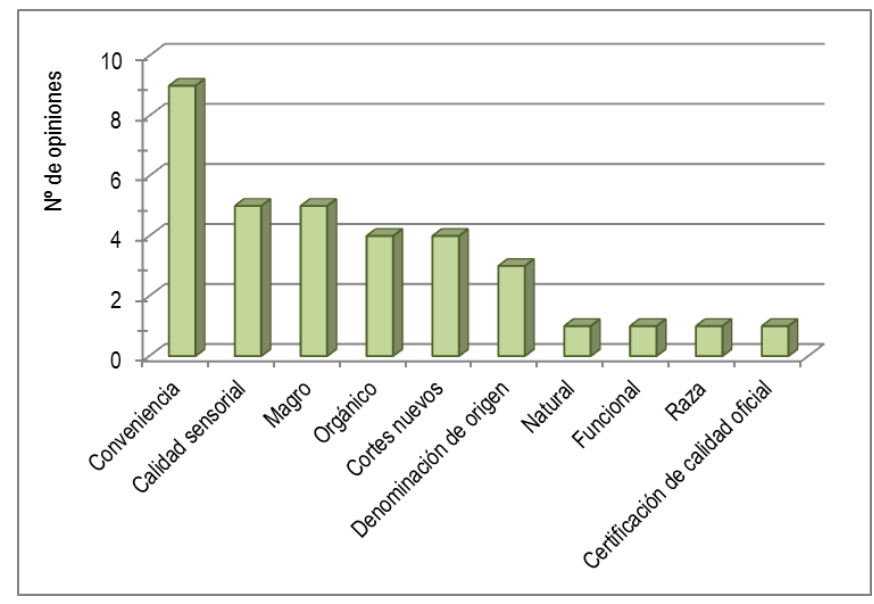

Se constató una interesante diferencia en las respuestas sectoriales. Los representantes del sector carnicería vieron más limitaciones al desarrollo de productos de alta calidad, debido al alto precio de estos productos y el bajo consumo relativo de carne bovina, probablemente debido a que la mayoría de sus sucursales se encuentran en sectores de menor poder adquisitivo. En el sector de los supermercados la preocupación es el bajo volumen de producción, insuficiente para suplir la demanda de los sectores de mayor ingreso.

\subsection{Estrategias para el desarrollo de carne bovina diferenciada}

Este estudio identificó que los atributos de calidad factibles de explotar en un corto plazo, por requerir menores cambios en la cadena de producción y existir un adecuado desarrollo tecnológico en plantas faenadoras y procesadoras, son nuevas presentaciones, carnes graduadas por calidad y carnes preparadas. Carnes magras o de calidad sensorial también tienen potencial, pero ofrecerlas requiere cambios tecnológicos ya en la producción primaria. La opción de nuevas presentaciones, en especial cortes o presentaciones utilizadas en otros países que ya forman parte de las líneas productivas de plantas procesadoras de carne para exportación, toma en cuenta que hay consumidores con experiencias culinarias en el extranjero y que la norma chilena permite ofrecer cortes de fantasía, sería posible aprovechar en nuestro mercado. Además, alternativas de despunte y de presentaciones para un mismo corte, podrían diferenciar un trozo de carne al momento de hacer la compra. Por ejemplo, la oferta de cortes con hueso y sus variaciones puede ser interesante al considerar la elegancia que se les asigna, su escasa presencia en nuestro mercado y la imposibilidad de importarlos desde los principales países abastecedores. El aprovechamiento de los estudios de perfil muscular, puede ser otra manera de agregar valor, ya que permite sugerir el uso de un corte según sus características, definir la terneza de las distintas partes de un músculo, optimizar la manera en que se realiza el desposte e incrementar la demanda y el valor de los cortes menos utilizados (Von Seggern y col., 2005; Calkins y col., 2005). Otra opción es desarrollar un sistema de graduación que permita al consumidor conocer la calidad de la carne al momento de compra. Una iniciativa como el sistema de clasificación de carnes desarrollado por el USDA permitiría diversificar la oferta de carne, acceder a un producto de calidad constante a través del tiempo y generar un gradiente de precios que favorecería la comercialización de todos los cortes provenientes de animales de mayor calidad. Con un sistema de este tipo y la debida información al público, el consumidor sería también capaz de apreciar productos como la carne de ternera o el lomo de buey, con el correspondiente beneficio que significa la agregación de valor de estas piezas. 
Se observó también, que a pesar del creciente interés por una alimentación saludable, la oferta de carne diferenciada por este atributo es escasa. Una opción es la carne natural, considerando que los sistemas pastoriles de nuestro país facilitan su obtención. Aunque se dice que este tipo de producción determina un producto final de menor palatabilidad, los paneles de evaluación sensorial difieren en sus resultados. Mientras unos estudios no detectaron diferencias entre carnes terminadas a pradera y enfeed-lot en relación a terneza y jugosidad (Mandell y col.,1998; Kerth y col.,2007) o sabor (Kerth y col.,2007), otros demostraron una menor aceptabilidad de las carnes obtenidas a pastoreo (Johnson y col.,1992; Schaake y col.,1993; Mandell y col.,1998; Williams, 2007), aunque esto podría ser revertido al suplementar o cambiar a una dieta concentrada antes del sacrificio (Ducket y col.,1993).Un sistema de graduación de carnes permite explotar los beneficios para la salud que otorga la baja cantidad de grasa de la carne de ternera (Johnson y col.,1992; Williams, 2007). Ahora bien, debe considerarse que se trata de atributos de confianza, por lo que deben comunicarse a través de señales de calidad concretas, como un sello o una marca. A esto hay que sumar la poca información que manejan los consumidores, por lo que la difusión de las propiedades de estos productos será fundamental para su éxito en el mercado interno. En las entrevistas, las cadenas de supermercados destacaron la necesidad de relaciones a largo plazo con sus proveedores y grandes volúmenes, ya que son pocos los abastecedores que por sí solos podrían cumplir dicha demanda.

Aunque el consumidor tradicional de productos convenientes se caracteriza por una menor preocupación por atributos como sabor, salud o tipo de proceso productivo, con el tiempo este formato ha ido conquistando segmentos más exigentes (Grunert y col., 2002). Esta expansión en el mercado de los productos convenientes, junto a la creciente escasez de espacio y tiempo dedicado a la cocina, haría de estos productos una alternativa interesante a explotar. La previa preparación de la carne, desde el aliñado hasta la cocción, parece despertar más interés entre los expertos que otros atributos asociados a la conveniencia. Sin embargo, la incierta demanda y la inversión inicial dificultan la inclusión de estos productos a las líneas de producción de plantas procesadoras de carne.

Los entrevistados mencionaron con frecuencia que el desarrollo de productos de alta calidad organoléptica tiene potencial de éxito en Chile, pero no sugirieron ninguna forma para lograrlo. Un protocolo de producción a lo largo de toda la cadena de valor parece ser la forma más viable de obtener productos de palatabilidad garantizada. Al parecer, es necesario que se genere confianza entre las distintas partes de la cadena, que los consumidores sean más informados y que exista un mayor progreso socio-económico, para orientarse hacia este tipo de iniciativas. Lo mismo ocurre con el madurado en seco. Los costos involucrados en la implementación de este sistema, la escasa población que lo conoce y valora y las dificultades en su almacenamiento a nivel minorista, dificultarían su inclusión en nuestro mercado.

Independiente del atributo a desarrollar, antes de desarrollar un producto, se recomienda estudiar en las preferencias del consumidor chileno, lo que implica no sólo preguntar qué tipo de productos quieren. Experiencias en el desarrollo de productos demuestran que cuando se les hace esta pregunta, tienden a sugerir mejoras en productos existentes (Grunert, 2006). Para entender en efecto a los consumidores, se han diseñado modelos que permiten conocer los mecanismos que determinan la decisión de compra, las tendencias en el desarrollo de los motivos de compra y el rol de factores situacionales (Grunert, 2006). Junto a estos productos es posible construir una marca, lo que da la posibilidad de distinguir los productos y crear fidelidad en los consumidores. Además, en una rutina de compra que dispone cada vez de menos tiempo, una marca bien desarrollada puede entregar una importante información en una señal de calidad de fácil reconocimiento (Bredahl, 2003). La marca eso sí, debe ser apoyada por estrategias promocionales que comuniquen las propuestas derivadas de la garantía de calidad que se ofrece (Verbeke y Viaene, 1999).

Junto con informar acerca de los atributos de un producto, se requiere ampliar el conocimiento del consumidor chileno acerca de las cualidades de la carne, así como los factores que determinan su calidad. La falta 
de información lleva a los consumidores a tomar decisiones equivocadas al momento de realizar la compra y en consecuencia una baja correspondencia entre la expectativa de calidad y la calidad obtenida al consumir el producto, lo que resulta en incertidumbre e insatisfacción (Brunsøy col., 2005). La gran mayoría de los entrevistados coincide con esto, afirmando que el primer paso a seguir para lograr el éxito de productos de calidad en nuestro mercado, es generar información y educación a los consumidores . Para que dicha iniciativa sea exitosa, probablemente será necesario apoyarla con una campaña publicitaria que derrumbe la percepción que tienen los consumidores respecto a los efectos de la carne bovina sobre la salud y destaque el valor nutricional de alternativas como las carnes magras. En la entrevista se preguntó a los expertos acerca del desarrollo de una campaña de fomento al consumo de carne bovina, en la que participe la totalidad de la cadena productiva. Dicha propuesta tuvo en general una recepción muy positiva, destacando la importancia de que se tome una medida de estas características. Sin embargo, coinciden en que sería muy difícil, principalmente debido a la atomización del sector, la competencia desleal entre sus participantes y el importante porcentaje de carne importada que participa en el mercado nacional. Otros destacan como necesario para el éxito de cualquier iniciativa, la coordinación de la cadena de la carne y el desarrollo de los productos en volumen suficiente y constante en el tiempo.

Figura 4: Actividades necesarias para el desarrollo de productos diferenciados en el mercado nacional según los expertos ( $n^{\circ}$ de opiniones).

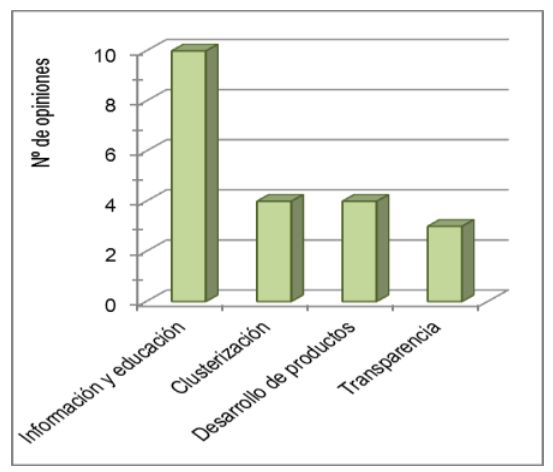

\section{Conclusiones}

Los resultados de este trabajo muestran que existe un incipiente desarrollo en la oferta de atributos de valor de los productos de carne bovina en el mercado minorista nacional. Sin embargo, en comparación a la de otros mercados, la oferta es reducida y puede sin duda ser ampliada. El análisis de las entrevistas a actores relevantes del sector, permitió identificar nuevas tendencias en la compra y consumo de carne, en especial entre los consumidores de mayor poder adquisitivo. Dichas tendencias son percibidas por los entrevistados como una oportunidad para desarrollar y posicionar productos con nuevos atributos de calidad en nuestro mercado. Sin embargo, el desarrollo de estos productos potencialmente exitosos, se ve enfrentado a grandes limitaciones asociadas, principalmente, al escaso conocimiento existente en nuestra sociedad respecto a la carne bovina. Para lograr el éxito de futuros productos especializados, es necesaria como primera medida, la generación de información y la educación de la población respecto a la carne bovina. Frente a este escenario, la propuesta de una campaña educativa que involucre la totalidad de la cadena productiva, tuvo una reacción positiva por parte de los entrevistados, pero reconocieron que sería difícil de realizar.

Aunque son necesarios estudios complementarios, entre los atributos potencialmente exitosos se pueden distinguir algunos que podrían llegar al consumidor nacional en un plazo más cercano. Entre estas alternativas se incluyen nuevas presentaciones, incluyendo cortes utilizados en el extranjero, alternativas de despunte y nuevas formas de exhibir un corte comercial. También es posible el desarrollo de un sistema de graduación de la carne, que genere un gradiente de precios y permita al consumidor acceder a distintas calidades de forma constante en el tiempo. Otra opción es la creación de una línea de carnes magras, obtenidas mediante sistemas a pastoreo o carne de ternera. Las carnes preparadas y productos de calidad sensorial superior obtenidos bajo sistemas de producción con exigentes protocolos productivos también son posibilidades de diferenciación. La aparición de nuevas preferencias por parte de los consumidores abre posibilidades de agregar valor $\mathrm{y}$ diferenciar la carne. Pero no debe olvidarse que entre 
más diferenciado es un producto, menor es la posibilidad de llegar a un gran grupo de personas, por lo que su desarrollo debe ir precedido del entendimiento de la demanda de los consumidores. La utilización de una marca que apoye cualquiera de los atributos a desarrollar es fundamental para la diferenciación de un producto y para la adecuada transmisión de sus atributos, muchas veces invisibles para el consumidor.

La escasa cantidad de información y conocimiento con que cuenta el consumidor de carne nacional, es la principal limitación al desarrollo de cualquier producto diferenciado. Por ello, es de primera necesidad desplegar estrategias promocionales, tanto a nivel público como empresarial. De esta manera será posible comunicar los beneficios nutritivos y los distintos atributos que puede ofrecer la carne como alimento. Los avances en la industria de la carne entregan una oportunidad para llenar los espacios que crean las nuevas demandas, pero existe gran desconfianza y atomización entre los actores de la cadena de la carne. Sólo depende de la coordinación de los interesados, el que las nuevas tendencias de compra sean aprovechadas como una ventaja competitiva.

\section{Referencia}

1. BECKER, T; 2000. Consumer perception of fresh meat quality: a framework for analysis. Brit Food J102 158-176.

2. BECKER, T.; BENNER, E.; GLITSCH, K. 2000. Consumer perception of fresh meat quality in Germany. Brit Food J102 246-266.

3. BERNUÉS, A.; OLAIZOLAB, A.; CORCORANC, K. 2003. Extrinsic attributes of red meat as indicators of quality in Europe: an application for market segmentation. Food QualPrefer 14 265-276.

4. BREDAHL, L. 2003. Cue utilization and quality perception with regard to branded beef. Food QualPrefer15 65-75.

5. BRUNS $\varnothing$, K.; BREDAHL, L.; GRUNERT, K.; SCHOLDERER' J. 2005. Consumer perception of the quality of beef resulting from various fattening regimes. Livest Prod Sci94 83-93.
6. CAB (Certified Angus Beef). 2010. Certified Angus

Beef.www.certifiedangusbeef.com/corp/integrity.p hp. [Consulta: 20-01-2010]

7. CALKINS, C.; JOHNSON, D.; GWARTNEY, B. 2005. El perfil muscular conlleva a mayor valor y demanda. Revista CarneTec. Enero-Febrero 2005 28-31.

8. CE (Comisión Europea). 2005. Food supply chains dynamics and quality certification. Institute for Prospective Technological Studies. Sevilla, España. $100 \mathrm{pp}$.

9. CE (Comisión Europea). 2007. European policy for quality agricultural products. Directorate-General for Agriculture and Rural Development. Bruselas, Bélgica.20 pp.

10. DUCKETT, S.K.; WAGNER, D.G.; YATES, L.D.; DOLEZAL, H.G.; MAY, S.G. 1993. Effects of time on feed on beef nutrient composition. J Anim Sci71 2079-2088.

11. FUNDACION CHILE. 2000. Cadena agroalimentaria de la carne bovina en Chile. Fundación Chile. Santiago, Chile. 68pp.

12. GRUNERT, K.; BAADSGAARD, A.; LARSEN, H.H.; MADSEN, T.K. 1996. Market orientation in food and agriculture. Kluwer Academic Publishers. Norwell, Massachusetts, Estados Unidos. 269 pp.

13. GRUNERT, K. 1997. What's in a steak? A crosscultural study on the quality perception of beef. Food QualPrefer 1 157-174.

14. GRUNERT, K.; BRUNS $\varnothing$, K.; FJORD, T. A. 2002. Consumers' food choice and quality perception. The Aarhus School of Business. Aarhus, Dinamarca. $60 \mathrm{pp}$.

15. GRUNERT, K.; BECH LARSEN T. 2004. Consumer perception of meat quality and implications for product development in the meat sector: a review. Meat Sci 66 259-272. 
16. GRUNERT, K. 2006. How changes in consumer behaviour and retailing affect competence requirements for food producers and processors. EconAgrRec Nat $\underline{6}$ 3-22.

17. JOHNSON, D.D; VAN HORN, H.H.; WEST, R.L.; HARRIS, B. Jr. 1992. Effect of calf management on carcass characteristics and palatability traits of veal calves. J Dairy Sci75 2799-2804.

18. KERTH, C.R.; BRADEN, K.W. COX, R. KERTH, L.K.; RANKINS, D.L. Jr. 2007. Carcass, sensory, fat color, and consumer acceptance characteristics of Angus-cross steers finished on ryegrass (Lolium multiflorum) forage or on a high-concentrate diet. Meat Sci75 324-331

19. LABEL VIANDE. 2010. Label Rouge. www.labelviande.com/fil-rouge-lexique-label-rouge.htm. [Consulta 12-01-2010].

20. LASTA, J. 2003. Terneza en la carne bovina. RevHereford. 6788-90.

21. LOUREIRO, M.L.; McCLASKEY, J.J. 2000. Assessing consumer response to Protected Geographical Identification labelling. AgriBus 16309-320.

22. MANDELL, I.B.; BUCHANAN-SMITH, J.G.; CAMPBELL, C.P. 1998. Effects of forage vs grain feeding on carcass characteristics, fatty acid composition, and beef quality in Limousin-cross steers when time on feed is controlled. J Anim Sci 762619-2630.

23. ODEPA (Oficina de Estudios y Políticas Agrarias). 2007. Carnes de ave y porcina lideraron el consumo de este rubro en los chilenos en 2006. www.odepa.gob.cl/odepaweb/jsp/odepad.jsp.

[Consulta: 16-03-2009]

24. OYARZUN, M.T. 2001. Sellos de calidad en los alimentos: el caso de la Unión Europea y Francia. FAO (Organización para los Alimentos y la Agricultura). Santiago, Chile. 11 pp.
25. PONCE, M. 2005. La industria de la carne en Chile 1955-2005. Puerto de Palos. Santiago de Chile, Chile. 281 pp.

26. PRIDE, W; FERREL, O. 1997. Marketing: conceptos y estrategias.Novena edición. Mc Graw Hill. Ciudad de México, México. 877 pp.

27. PROCOM (Programa Pro Competitividad). 2004. Carne bovina: Desafíos y potencial exportador. Santiago, Chile. PROCOM. SOFOFA (Sociedad de Fomento Fabril), AMCHAM Chile (Cámara Chileno Norteamericana de Comercio), CORFO (Corporación de Fomento de la Producción), McKinsey\&Company. 70pp.

28. RKM (Center for Research \& Knowledge Management). 2010. http://www.beefresearch.org/CMDocs/BeefResear ch/Aging\%20Overview.pdf. [Consulta 26-032010].

29. SAG (Servicio Agrícola y Ganadero) - IICA (Instituto Interamericano de Cooperación para la Agricultura).1999. Ganadería bovina y ovina: visiones para una discusión. Santiago, Chile. SAG, IICA. 102 pp.

30. SAMPIERI, R.; FERNANDEZ, C.; BAPTISTA, P. 2006.Metodología de la investigación.McGraw Hill.4ª Ed. México. Pp 581-682.

31. SAVELL, J. 2008. Dry-aging of beef: Executive Summary. Center for Research and Knowledge Management. National Cattlemen's Beef Association. Texas, Estados Unidos. 14 pp.

32. SCHAAKE, S.L.; SKELLEY, G.C.; HALPIN, E.; GRIMES, L.W.; BROWN, R.B.; CROSS, L.; THOMPSON, C.E. 1993. Carcass and meat sensory traits of steers finished on fescue and clover, summer forage, or for different periods in drylot. $\mathbf{J}$ Anim Sci. 713199-3205.

33. SMITH, G.C.; TATUM, J.D.; BELK, K.E.; SCANGA, J.A. 2008. Post-Harvest Practices for Enhancing Beef Tenderness. Center for Research and Knowledge Management. National 
Cattlemen's Beef Association. Colorado, Estados Unidos. 20 pp.

34. TATUM, J.D.; BELK, K. E.; GEORGE, M. H.; SMITH, G. C. 1999. Identification of quality management practices to reduce the incidence of retail beef tenderness problems: development and evaluation of a prototype quality system to produce tender beef. J Anim Sci 772112-2118

35. TERNERA ASTURIANA. 2010.Ternera asturiana. www.terneraasturiana.org/gestion.html. [Consulta 15-03-2010].

36. VON SEGGERN, D.;CALKINS, C.R.; JOHNSON, D.D.; BRICKLER, J.E.; GWARTNEY, B.L. 2005. Muscle profiling: Characterizing the muscles of the beef chuck and round. Meat Sci7139-51

37. VERBEKE, W.; VIAENE, J. 1999. Consumer attitude to beef quality labelling and associations with beef quality labels. JInt Food AgriBus Market10 45-65.

38. WILLIAMS, P.G. 2007. Nutritional composition of red meat. NutrDietet 64 S113-S119. 